\title{
Technical Note: The determination of enclosed water volume in large flexible-wall mesocosms "KOSMOS"
}

\author{
J. Czerny, K. G. Schulz, S. A. Krug, A. Ludwig, and U. Riebesell \\ GEOMAR - Helmholtz Centre for Ocean Research Kiel, 24105 Kiel, Germany \\ Correspondence to: J. Czerny (jczerny@geomar.de)
}

Received: 29 August 2012 - Published in Biogeosciences Discuss.: 19 September 2012

Revised: 28 January 2013 - Accepted: 3 February 2013 - Published: 20 March 2013

\begin{abstract}
The volume of water enclosed inside flexible-wall mesocosm bags is hard to estimate using geometrical calculations and can be strongly variable among bags of the same dimensions. Here we present a method for precise water volume determination in mesocosms using salinity as a tracer. Knowledge of the precise volume of water enclosed allows establishment of exactly planned treatment concentrations and calculation of elemental budgets.
\end{abstract}

\section{Introduction}

Manipulation of a chemical parameter (e.g. nutrient or pollutant) in an experimental enclosure is usually accomplished by the following: (1) calculating the amount of the substance needed for a given volume of water, (2) adding the substance, (3) mixing the enclosed water to ensure homogeneity and (4) analysing the water to check if correct concentrations are achieved. In a large pelagic mesocosm like KOSMOS (Kiel Offshore Mesocosms for future Ocean Simulations) ( $~ 50$ $80 \mathrm{~m}^{3}$ within each unit), some of these steps are technically difficult. The precise volume of water cannot be easily measured using standard volumetric or gravimetric methods and, as shown in this article, geometric calculations do not deliver satisfying results for a flexible-wall enclosure. Distributing a substance within an up to 25 -m-deep water column can lead to vertical concentration gradients, and active mixing requires a large energy input. When a sample is analysed, it might be already too late to detect applied treatment concentrations inside the mesocosm as they might be rapidly altered by biological activity. Uncertainties and variability in treatment levels and budget calculations can be largely avoided if the exact water volumes of individual mesocosms are known.
Many chemical parameters can be adjusted much more precisely as they can be determined later using seawater analytics. Here we present a method to precisely measure the volume within each experimental unit by addition of relatively small amounts of sodium chloride solution. Errors and uncertainties of the volume measurement are discussed.

\section{Preparation of salt brine for volume measurements}

Sodium chloride $(\mathrm{NaCl})$ is used as a conductometric tracer for volume measurement because of its high solubility $\left(359 \mathrm{~g} \mathrm{~L}^{-1}\right.$ at $\left.20^{\circ} \mathrm{C}\right)$. Complex sea salt mixtures cannot be prepared due to the relatively low solubility of some of the components. A $\mathrm{NaCl}$ concentration well below saturation (i.e. $250-300 \mathrm{~g} \mathrm{~kg}^{-1}$ ) was chosen to ensure relatively quick dissolution and to prevent possible precipitation which could bias volume measurement. The source of the salt should be chosen with care, as impurities such as iron can cause enrichment of the enclosed water far beyond natural levels. To prevent this, one option is to use high purity grade salt. However, other salts are relatively pure and cost efficient depending on the production process. Attention should be paid to choosing a salt which does not contain commonly used anti-caking agents such as ferocyanide (e.g. Brezelsalz Bäckerstolz ${ }^{\circledR}$, esco, Germany). The ion exchanger Lewatit ${ }^{\mathrm{TM}}$ MonoPlus TP $260^{\circledR}$ (Lanxess, Germany) removes metal anions efficiently from concentrated salt solutions. As soon as a subsample for calibration is taken after complete dissolution, the brine has to be stored in a tightly closed container to avoid evaporation. For a schematic drawing of our setup to prepare salt brine, see Fig. 1. 


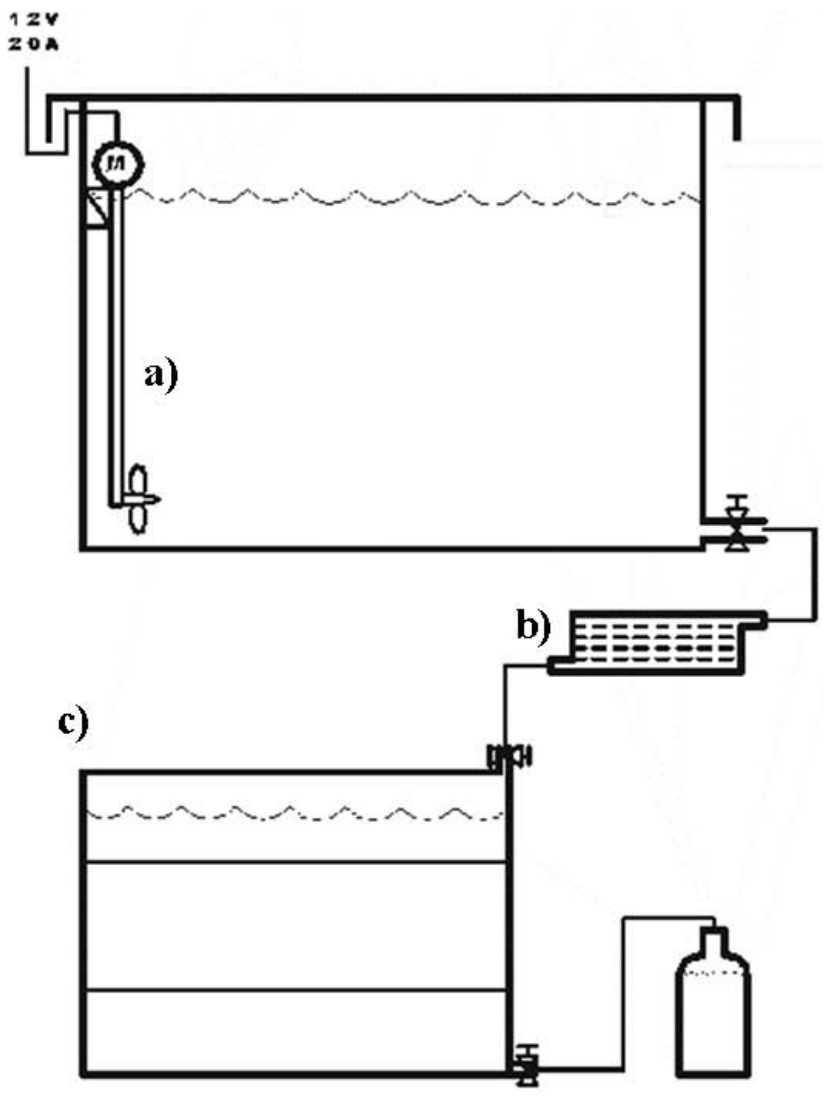

Fig. 1. Schematic drawing of the setup used for the preparation of salt brine in large amounts. An electric outboard motor (a) in the mixing tank speeds up the dissolution of salts, enabling preparation of nearly saturated solutions within a few hours. Produced solutions can be further processed (e.g. run over filters or purifying columns) (b) and stored in intermediate bulk containers (c) and dispensed into suitable carboys for quantitative addition into the mesocosms using the "spider".

\section{Salt brine addition}

Prior to brine addition the initial salinity in the mesocosms has to be determined precisely. Due to the slightly uneven shape of the enclosures, mean salinity of a CTD (conductivity, temperature and depth) profile made in a stratified mesocosm is not necessarily matching mean salinity of the same water without a salinity gradient (Fig. 2a). Therefore, the mesocosm water column has to be mixed prior to measurement until homogeneity is reached (if a salinity gradient is found). Mixing was performed by bubbling with compressed air for five minutes via a weighted $(\sim 13 \mathrm{~mm}$ inner diameter) hose lowered to the bottom of the mesocosm. After determination of initial salinity by three consecutive CTD profiles, a precisely weighted amount of salt brine was injected into each mesocosm. Amounts of brine added were calculated based on an approximated volume to achieve salinity increases of 0.2 to 0.4 . The "spider" system (described by
Riebesell et al., 2012) was designed to evenly distribute liquids of any density inside large mesocosms. The brine solution was pumped through the "spider" system which was continuously moved up and down the upper $\sim 90 \%$ of the mesocosm depth, avoiding any resting of the device at the lowest point. Once the brine solution has been pumped into the mesocosms, the empty solution container was rinsed twice with mesocosm water and the remaining water was pressed out of the "spider" using compressed air. The established salt gradient with decreasing water density with depth is not stable. An overturning circulation mixes the water column during the following $12 \mathrm{~h}$ as light, low-salinity water is making its way up across the denser, high-salinity water sinking to the bottom (Fig. 2b). While temperature gradients establish within hours according to outside stratification, salinity distribution remains homogeneous throughout the procedure. A stable halocline can be later established by purposeful salt brine addition to deep water layers or by freshwater input to the surface.

\section{Salinity measurement}

Salinity profiles were collected using a data-loggerequipped, hand-held, multisensory CTD CTD60M (Sun and Sea Technologies), manually lowered at a speed of $\sim 0.2 \mathrm{~m} \mathrm{~s}^{-1}$. Reproducibility of mean salinity (standard deviation of mean salinity from three replicate profiles $(n=\sim$ 300 single measurements per profile) in four salinity measurements) was typically $\leq 0.0003$ units. This is corresponding to a measurement-derived uncertainty for volume estimates of $\sim 0.1 \%$ for a salt addition increasing salinity $(S)$ by 0.3 .

\section{Calibration}

The calibration of the salt brine was performed at $20^{\circ} \mathrm{C}$ in the laboratory, using surface water collected in the Flensburg Fjord $(S=17.0)$. Nine different mixing ratios were measured to construct a calibration curve (Fig. 3). For this, seawater has to be stirred in an appropriate calibration beaker to establish a constant flow across the salinity sensor of the CTD probe for a stable reading. Determination of initial salinity in the calibration beaker was followed by a first addition of brine, oriented at the largest expected mesocosm volume (highest dilution). Afterwards salinity was increased in small steps until the mixing ratio of the smallest expected mesocosm volume was reached. After plotting the first calibration curve, the batch of water was mixed again and a second calibration starting at $S=17.1$ was performed.

Although conductivity was increased by $\mathrm{NaCl}$ addition and not using a complex sea salt mixture, measured increases in salinity were directly proportional to the added amount of salt brine. The algorithm used by our CTD (UNESCO PSS78) assumes seawater ion composition to be conservative 

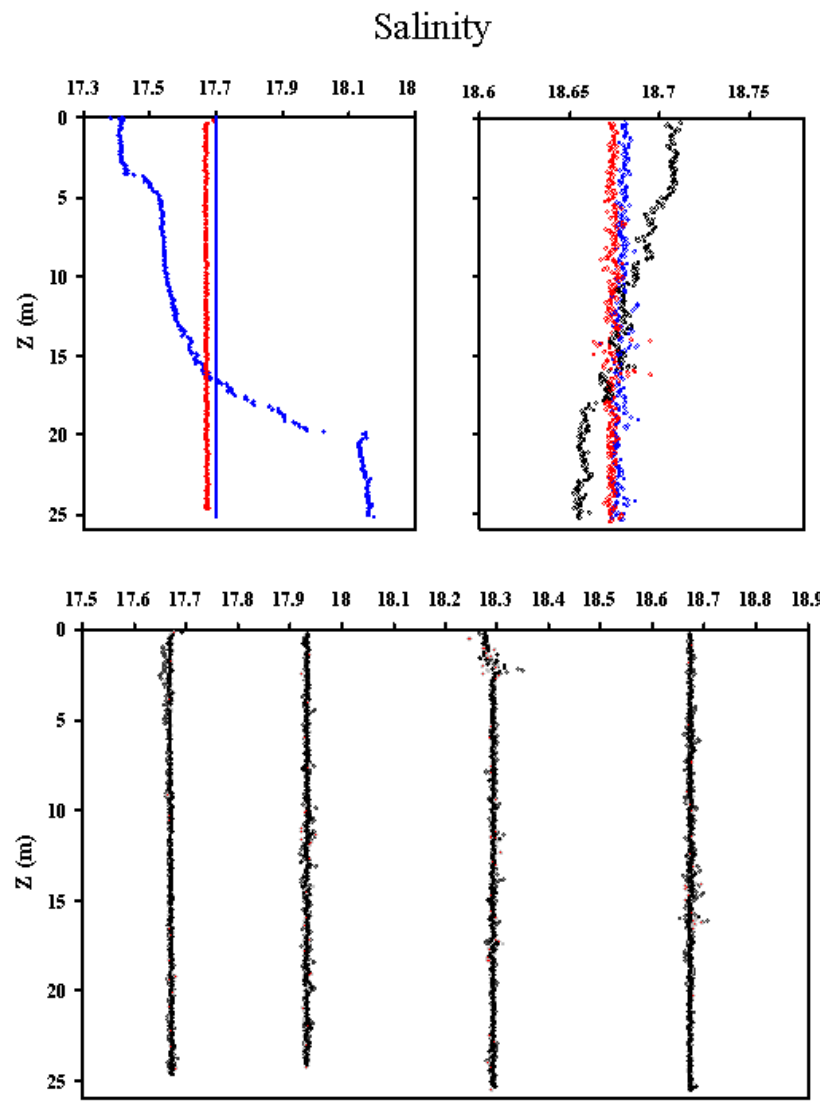

Fig. 2. (a) A natural vertical salinity profile in a 25-m-deep mesocosm (blue dots). The average salinity of the profile is indicated by a vertical blue line. Red dots are salinity measurements after mixing the water column using 5-min bubbling with compressed air. Differences are caused by the uneven shape of the bag (here especially the bottom funnel) (b) The black profile is collected right after injecting salt brine to the upper $22 \mathrm{~m}$ of the mesocosm, the blue profile is measured $6 \mathrm{~h}$ later and the red profile $18 \mathrm{~h}$ later. (c) Homogeneous $S$ profile shown in (a) is increased in three steps, measured on three consecutive days using three replicated profiles shown as black, red and gray dots.

when calculating salinity from conductivity and temperature. However, changes in sea salt composition were found to have no significant influence on volume determination using this protocol.

When the gravimetric mixing ratio of seawater in the beaker per added brine ( $\mathrm{SW}$ : Brine $(\mathrm{kg})$ ) is plotted versus measured salinity increase $(\Delta S)$, a power fit can be used to calculate volumes of mesocosms from $\Delta S(X)$ by multiplication of the mixing ratio $(Y)$ with the added mass of brine (Fig. 3). To determine the precision of volume measurements, three consecutive salt additions were performed in a 25-m-deep mesocosm on a test cruise in the Flensburg Fjord in the western Baltic Sea in January 2011 (Fig. 2c).

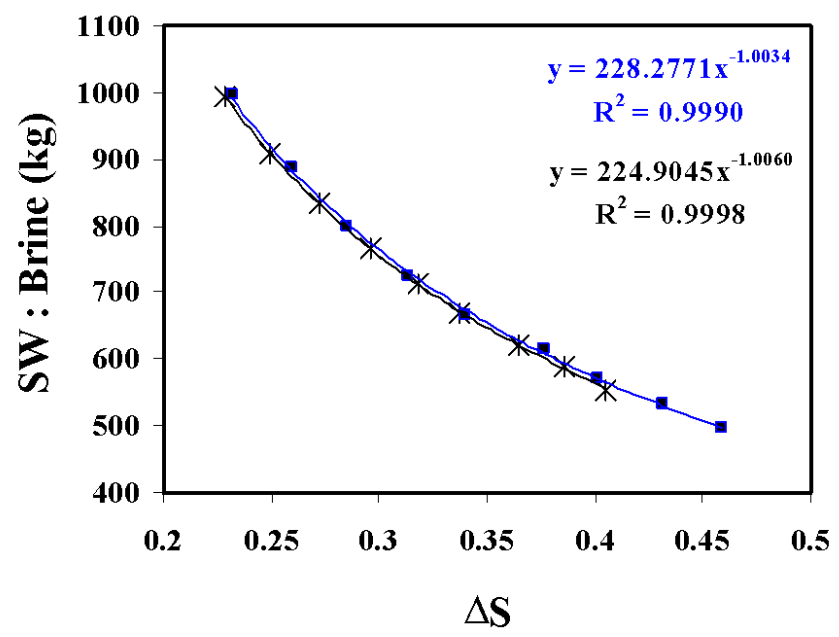

Fig. 3. Salt calibration curve. Exponential fits for two calibration datasets: calibration 1 (blue curve) is measured by increasing salinity from natural Baltic seawater $S=17$; calibration 2 (black curve) is done after salinity was already increased from 17 to 17.1 by $\mathrm{NaCl}$ addition. Power formulas can be used to calculate mesocosm volume by multiplying $y$ with the added amount of brine.

\section{Potential side-effects of volume measurements using $\mathrm{NaCl}$ as a tracer}

Every perturbation of the enclosed ecosystem in a mesocosm bares the risk of biasing results and reducing comparability to the natural system. Experiments on enclosed ecosystems cannot, however, be done without significant changes to natural physical and chemical side parameters such as turbulence, light or artificial nutrient additions. Perturbations equally performed in all parallel mesocosms are unproblematic when effects are compared among treatments. Homogenisation of the initial stratification and the addition of salt are equally performed in all units. Bubbles used to mix the mesocosms in the beginning of the experiment are presumably stressful for some of the enclosed organisms and might change initial dissolved gas concentration. Initial mixing of the water column in the beginning of the experiment has, beside the measurement of the salt inventory, a further advantage: it equalises differences in vertical nutrient distribution among parallel treatments arising from partial disruption of stratification after closing of the mesocosms. Harmful effects of temporary short bubbling on gases or organisms were not observed in prior experiments. In order to minimise stress on the enclosed ecosystem, the mesocosms should be mixed with large bubbles and not longer than absolutely necessary. Reports on physiological responses to a salinity increase of less than 1 could not be found for marine organisms. A minimum in species richness at intermediate salinities is observed along the natural salinity gradient $(0$ to 34) of the Baltic Sea estuary. However, changes in species distribution are generally attributed to salinity changes of 
Table 1. Volumes calculated for three salinity additions to the same mesocosm using two calibrations, Cal 1 and Cal 2, were applied to each individual salinity increase. Results were corrected for increasing volume due to the addition of brine.

\begin{tabular}{lrrrr}
\hline & & & \multicolumn{2}{c}{$\begin{array}{c}\text { Mesocosm Volume } \\
\text { Estimates (t) }\end{array}$} \\
\cline { 3 - 5 } Addition & $\begin{array}{r}\text { Added } \\
\text { Nr. }\end{array}$ & $\Delta S$ & Cal 1 & Cal 2 \\
& brine (kg) & & & \\
\hline 1 & 83.05 & 0.264 & 72.44 & 71.1 \\
2 & 113.16 & 0.359 & 72.40 & 71.1 \\
3 & 122.27 & 0.381 & 73.53 & 72.2 \\
\hline Mean & & & 72.79 & 71.5 \\
St. Dev. \% & & & 0.88 & 0.89 \\
\hline
\end{tabular}

several units with responses below 0.5 not being resolved (Remane, 1934; Paavola et al., 2005). Potential impacts of $\mathrm{NaCl}$ on freshwater ecosystems are summarised by Siegel (2007) within a risk assessment for salt use on roads. Responses to prolonged exposure are generally observed when several $\mathrm{g} \mathrm{kg}^{-1} \mathrm{NaCl}$ were added. Single studies reported effects on Cladocera and some fish juveniles already at about $0.5 \mathrm{~g} \mathrm{~kg}^{-1}$ of salt addition (see table and references within Siegel, 2007). Thus, $\mathrm{NaCl}$ could be generally also used as a tracer in freshwater mesocosm research if salt additions are below $0.2 \mathrm{~g} \mathrm{~kg}^{-1}$. In any case, the risk of biasing results by additional manipulation of the enclosed system has to be evaluated against advantages of knowing the precise volume of the enclosure.

\section{Discussion of measurement errors}

Volumes calculated from three consecutive salt additions to the test mesocosm are summarised in Table 1. Deviations of about $\pm 1 \%$ are larger than expected from possible uncertainties in the amount of brine added and the precision of salinity measurements. Based on the reproducibility of measurements we would expect uncertainties of only up to $\pm 0.1 \%$. With respect to the good reproducibility of salinity measurements inside the mesocosms, enrichments of 0.3 to 0.4 during the Baltic Sea test turned out to be unnecessarily high. Comparable results could have been achieved applying salinity increases of less than 0.1. Actual losses during addition of brine can be expected to be on the order of single grams. Observed deviations are therefore unlikely to be caused by addition or measurement errors inside the mesocosms, but from uncertainties arising from the calibration. Calculated volumes based on three salinity additions to the same mesocosm and two calibration curves were used to identify uncertainties. Using either calibration 1 or 2 , results of the consecutive measurements vary by the same percentage $(\sim 0.9 \%)$; however, using calibration 1 , mean volume is $1.2 \%$ higher
Table 2. Measured volume of nine mesocosms in two experiments: Bergen 2011 and Svalbard 2010 including maximum and standard deviations from mean measured volumes. In Bergen 2011, bags reached overall $25 \mathrm{~m}$ below the surface, geometrically calculated volume for the bags, funnel shaped in the bottom $2 \mathrm{~m}$, is $74.3 \mathrm{t}$. In Svalbard 2010, cylindrical 15-m-deep bags are geometrically calculated to hold $47 \mathrm{t}$.

\begin{tabular}{lrr}
\hline $\begin{array}{l}\text { Mesocosm. } \\
\text { Nr. }\end{array}$ & Bergen & $\begin{array}{r}\text { Svalbard } \\
2011\end{array}$ \\
\hline 1 & 76.8 & 48.8 \\
2 & 79.9 & 48.1 \\
3 & 78.4 & 46.7 \\
4 & 73.5 & 48.7 \\
5 & 75.9 & 46.5 \\
6 & 73.5 & 47.2 \\
7 & 79.4 & 48.8 \\
8 & 78.4 & 45.0 \\
9 & 75.2 & 47.8 \\
\hline Max. Dev. \% & 8.4 & 7.9 \\
St. Dev. \% & 3.2 & 2.7 \\
\hline
\end{tabular}

than volume calculated using calibration 2 . This offset is obviously due to a $\sim 0.003$ uncertainty in calibration initial $S$, to which all $\Delta S$ values in the calibration curve are referenced to. Parts of the calibration limitation might have been due to problems in measuring salinity with a CTD probe inside a beaker. Consequently, the method is more sensitive in determining differences between mesocosms than in determining the absolute amount of water enclosed. Most accurate results can be expected when calibration is done using seawater at in situ $T$ and $S$, and the initial salinity is repeatedly measured.

\section{Observed variability between mesocosms}

Despite their nearly cylindrical appearance, measured volumes of nine KOSMOS mesocosms in two experiments deviated by up to $8 \%$ between parallel units (standard deviation of $\sim \pm 3 \%$; Table 2). The 25-m-deep setup in the Bergen 2011 experiment had slightly larger deviations than the 15m-deep setup used in Svalbard 2010. The volumes of the 25m-long bags were $4 \%$ smaller than their geometrically calculated volumes during the test in the Baltic Sea. In Bergen 2011 the volumes of the same bags were averaged 3.3\% larger than geometrically calculated. These differences were probably caused by differences during filling - especially opening time - changing water densities, and slight lateral deformations caused by water currents acting on the moored mesocosms. During an earlier test cruise more than $20 \%$ variation was measured between three mesocosms filled at a relatively strong current. 
Acknowledgements. This work is a contribution to the "European Project on Ocean Acidification" (EPOCA) which received funding from the European Community's Seventh Framework Programme (FP7/2007-2013) under grant agreement no. 211384. Financial support was provided through Transnational Access funds by the European Union Seventh Framework Program (FP7/2007-2013) under grant agreement no. 22822 MESOAQUA and by the Federal Ministry of Education and Research (BMBF, FKZ 03F0608) through the BIOACID (Biological Impacts of Ocean ACIDification) project. We gratefully acknowledge the logistical support of Greenpeace International for assistance with the transport of the mesocosm facility from Kiel to Ny-Ålesund and back. We also thank the captains and crews of M/V ESPERANZA of Greenpeace, $\mathrm{R} / \mathrm{V}$ Viking Explorer of the University Centre in Svalbard (UNIS) and R/V Alkor for assistance during mesocosm transport and during deployment and recovery in Kongsfjorden and the Flensburg fjord, respectively. We thank the staff of the French-German Arctic Research Base at Ny-Ålesund, in particular Marcus Schumacher, for on-site logistical support.

The service charges for this open access publication have been covered by a Research Centre of the Helmholtz Association.

Edited by: T. F. Thingstad

\section{References}

Paavola, M., Olenin, S., and Leppakoski, E.: Are invasive species most successful in habitats of low native species richness across European brackish water seas?, Estuar. Coast. Shelf Sci., 64, 738-750, doi:10.1016/j.ecss.2005.03.021, 2005.

Remane, A.: Die Brackwasserfauna, Verh. Deut. Z., 36, 34-74, 1934.

Riebesell, U., Czerny, J., von Bröckel, K., Boxhammer, T., Büdenbender, J., Deckelnick, M., Fischer, M., Hoffmann, D., Krug, S. A., Lentz, U., Ludwig, A., Muche, R., and Schulz, K. G.: Technical Note: A mobile sea-going mesocosm system - new opportunities for ocean change research, Biogeosciences Discuss., 9, 12985-13017, doi:10.5194/bgd-9-12985-2012, 2012.

Siegel, L.: Hazard identification for human and ecological effects of sodium chloride road salt Ph.D. theses, PE, State of New Hampshire, Department of environmental services, Water division, Watershed management, 2007. 